\title{
Injective Modules and Prime Ideals of Universal Enveloping Algebras
}

\author{
Jörg Feldvoss* \\ Department of Mathematics and Statistics \\ University of South Alabama \\ Mobile, AL 36688-0002, USA \\ Dedicated to Professor Edgar E. Enochs on the occasion of his 72nd birthday
}

\begin{abstract}
In this paper we study injective modules over universal enveloping algebras of finite-dimensional Lie algebras over fields of arbitrary characteristic. Most of our results are dealing with fields of prime characteristic but we also elaborate on some of their analogues for solvable Lie algebras over fields of characteristic zero. It turns out that analogous results in both cases are often quite similar and resemble those familiar from commutative ring theory.
\end{abstract}

2000 Mathematics Subject Classification: 17B35, 17B50, 17B55, 17B56

\section{Introduction}

In this paper we investigate the injective modules and their relation to prime ideals in universal enveloping algebras of finite-dimensional Lie algebras. Especially, in the case that the ground field is of prime characteristic we obtain several results that seem to be new. It should be remarked that most of the results of the first two sections and the last section are already contained in an unpublished manuscript of the author (cf. [Fel90] ) but the entire third section and Theorem 4.3 are completely new. In the following we will describe the contents of the paper in more detail.

The first section provides the framework for the paper. We begin by recalling the well-known result from noetherian ring theory that every injective module decomposes uniquely (up to isomorphism and order of occurrence) into a direct sum of indecomposable injective modules. Then it is shown that universal enveloping algebras of finite-dimensional Lie algebras over fields of prime characteristic are FBN rings. As a consequence, indecomposable injective modules are in bijection with prime ideals. Moreover, it is proved that the universal enveloping algebra of a finite-dimensional Lie algebra over a field of prime characteristic is a Matlis ring (i.e., every indecomposable injective module is the injective hull of a prime factor ring of the universal enveloping algebra considered as a one-sided module) if and only if the underlying Lie algebra is abelian. A similar result might also hold in characteristic zero but we were neither able to prove this nor to find it in the literature.

In the second section we study certain finiteness conditions for injective hulls. It is well-known from a result obtained by Donkin Don82 and independently by Dahlberg Dah84 that injective hulls of locally finite modules over universal enveloping algebras of finite-dimensional solvable Lie

${ }^{*}$ E-mail address: jfeldvoss@jaguar1.usouthal.edu 
algebras over fields of characteristic zero are again locally finite. We show that the converse of this result holds, i.e., the locally finiteness of injective hulls of locally finite modules in characteristic zero implies that the underlying Lie algebra is solvable. In fact, the locally finiteness of the injective hull of the one-dimensional trivial module already implies that the underlying Lie algebra is solvable. This generalizes an observation of Donkin in Don82. Moreover, we prove that every essential extension of a locally finite module over a universal enveloping algebra of any finite-dimensional Lie algebra over a field of prime characteristic is locally finite by applying a result of Jategaonkar Jat74 in conjunction with the result from the first section saying that universal enveloping algebras of finite-dimensional Lie algebras over fields of prime characteristic are FBN rings. In particular, injective hulls of locally finite modules are always locally finite. By generalizing slightly another result of Jategaonkar Jat75, we also show that injective hulls of artinian modules over universal enveloping algebras of finite-dimensional Lie algebras over a field of prime characteristic are always artinian. Finally, it is established that for the universal enveloping algebra of a finite-dimensional Lie algebra over a field of prime characteristic non-zero noetherian modules are never injective by proving that the injective dimension of a noetherian module coincides with the dimension of the underlying Lie algebra. On the other hand, there are artinian and locally finite modules of any possible injective dimension.

In the third section we consider certain locally finite submodules of the linear dual of a universal enveloping algebra. We start off by showing how an argument from Dah84 can be changed slightly to make it work over arbitrary fields of any characteristic and therefore obtaining a different (and in our opinion more transparent) proof of a result due to Levasseur [Lev76. Then we give a very short proof of the main result of Kos54 by using the locally finiteness of injective hulls of locally finite modules over universal enveloping algebras of finite-dimensional solvable Lie algebras in characteristic zero in an essential way. In fact, this argument was motivated by our proof of the injectivity of the continuous dual of the universal enveloping algebra of an arbitrary finite-dimensional Lie algebra over a field of prime characteristic. As an immediate consequence, we obtain that in prime characteristic the cohomology with values in the continuous dual vanishes in every positive degree. In particular, Koszul's cohomological vanishing theorem does remain valid in prime characteristic. These results seem to be new. Moreover, the modular cohomological vanishing theorem is much stronger than its analogue in characteristic zero which follows from a recent result of Schneider (cf. [Mas00) and says that the cohomology with values in the continuous dual vanishes in degrees one and two.

The last section closes the circle of ideas by coming back to the correspondence between injective modules and prime ideals. It is verified that universal enveloping algebras of finite-dimensional Lie algebras over fields of prime characteristic are injectively homogeneous in the sense of [BH88. As a consequence of the general theory of injectively homogeneous rings developed in [BH88] we obtain a nice description of a minimal injective resolution of the universal enveloping algebra as a module over itself in terms of the injective hulls of its prime factor rings considered as one-sided modules. In particular, this enables us to show that the last term of such a minimal injective resolution is isomorphic to the continuous dual which was proved by Barou and Malliavin [BM85] for finite-dimensional solvable Lie algebras over algebraically fields of characteristic zero.

Throughout this paper we will assume that all associative rings have a unity element and that all modules over associative rings are unital.

\section{Injective Modules and Prime Ideals}

Since the universal enveloping algebra of a finite-dimensional Lie algebra is left and right noetherian (cf. Jac79, Theorem V.6]), finding all injective left and right $U(\mathfrak{a})$-modules reduces to the clas- 
sification of the indecomposable ones (see Mat58, Theorem 2.5, Proposition 2.6, and Proposition 2.7]):

Proposition 1.1 Let $\mathfrak{a}$ be a finite-dimensional Lie algebra over an arbitrary field. Then the following statements hold:

(1) Every injective left or right $U(\mathfrak{a})$-module is a direct sum of indecomposable injective submodules.

(2) If I is an indecomposable injective left or right $U(\mathfrak{a})$-module, then $\operatorname{End}_{\mathfrak{a}}(I)$ is local. In particular, the decomposition in the first part is unique up to isomorphism and order of occurrence of the direct summands.

In order to be able to parameterize the indecomposable injective left or right $U(\mathfrak{a})$-modules, one needs the following concept from non-commutative ring theory. A left and right noetherian associative ring $R$ is called a $F B N$ ring if every essential left ideal and every essential right ideal of every prime factor ring of $R$ contains a non-zero two-sided ideal (which, in fact, is essential). While classifying the indecomposable injective $U(\mathfrak{a})$-modules by analogy with the commutative case (see Mat58, Proposition 3.1]), one should be aware that the injective hull of $U(\mathfrak{a}) / \mathcal{P}$ (considered as a left or right $U(\mathfrak{a})$-module) is not necessarily indecomposable for every prime ideal $\mathcal{P}$ of $U(\mathfrak{a})$. For example, the injective hull of $U(\mathfrak{a}) / \operatorname{Ann}_{U(\mathfrak{a})}(S)$ is isomorphic to the direct sum of $d$ copies of the injective hull of any simple a-module $S$ of dimension $d>1$ (cf. the proof of Theorem 1.3 and Theorem 4.3).

Let $M$ be a non-zero $U(\mathfrak{a})$-module. A two-sided ideal $\mathcal{P}$ is said to be associated to $M$ if there exists a submodule $N$ of $M$ such that $\mathcal{P}$ equals the annihilator of every non-zero submodule of $N$. It is well-known that $\mathcal{P}$ is necessarily prime and that for an indecomposable injective module $I$ there exists a unique prime ideal $\mathcal{P}_{I}$ associated to $I$ (cf. BGR73).

If $\mathfrak{a}$ is a finite-dimensional Lie algebra over a field of prime characteristic, then $U(\mathfrak{a})$ is a finitely generated $C(U(\mathfrak{a}))$-module (cf. [SF88, Theorem 5.1.2]). Hence one has the following well-known facts which are crucial for the results obtained in this paper:

(IC) $U(\mathfrak{a})$ is integral over its center $C(U(\mathfrak{a})$ ) (cf. [SF88, Theorem 6.1.4]). More generally, there exists a subalgebra $\mathcal{O}(\mathfrak{a}) \cong \mathbb{F}\left[t_{1}, \ldots, t_{\operatorname{dim}_{\mathbb{F}}}\right]$ of $C(U(\mathfrak{a}))$ such that $U(\mathfrak{a})$ is integral over every subring $C$ of $U(\mathfrak{a})$ with $\mathcal{O}(\mathfrak{a}) \subseteq C \subseteq C(U(\mathfrak{a}))$.

(PI) $U(\mathfrak{a})$ is a PI ring (cf. GW89, p. xi]).

The next result shows that the indecomposable injective modules over universal enveloping algebras in prime characteristic can be classified by their associated prime ideals.

Theorem 1.2 Let $\mathfrak{a}$ be a finite-dimensional Lie algebra over a field of prime characteristic. Then the universal enveloping algebra $U(\mathfrak{a})$ is a FBN ring. In particular, there is a one-to-one correspondence between the indecomposable injective $U(\mathfrak{a})$-modules and the prime ideals of $U(\mathfrak{a})$ given by $I \mapsto \mathcal{P}_{I}$, where $\mathcal{P}_{I}$ is the unique prime ideal associated to $I$.

Proof. The first assertion follows from GW89 Proposition 8.1(b)] and the second assertion is a consequence of the first and Kra72, Theorem 3.5].

Question. Let $\mathfrak{a}$ be a finite-dimensional Lie algebra over a field of characteristic zero. It would be interesting to know when $U(\mathfrak{a})$ is a FBN ring? Is $U(\mathfrak{a})$ only an FBN ring if $\mathfrak{a}$ is abelian?

An associative ring $R$ is called a Matlis ring if every indecomposable injective left or right $R$-module is isomorphic to the injective hull of $R / \mathcal{P}$ (considered as a left or right $R$-module, respectively) for some prime ideal $\mathcal{P}$ of $R$. Every left and right noetherian Matlis ring is a FBN ring (see [Kra72, Corollary 3.6]), but the converse is not true as follows from Theorem 1.2 and the next result. 
Theorem 1.3 Let $\mathfrak{a}$ be a finite-dimensional Lie algebra over a field of prime characteristic. Then the universal enveloping algebra $U(\mathfrak{a})$ is a Matlis ring if and only if $\mathfrak{a}$ is abelian.

Proof. Since both conditions are independent of the ground field $\mathbb{F}$, we can assume that $\mathbb{F}$ is algebraically closed. Suppose that $U(\mathfrak{a})$ is a Matlis ring. According to Kra70 Corollary 14], every prime ideal of $U(\mathfrak{a})$ is completely prime. Let $S$ be a simple $\mathfrak{a}$-module and set $\mathbb{D}:=\operatorname{End}_{\mathbb{F}}(S)$. Since $S$ is finite-dimensional (cf. $[\mathrm{SF} 88$, Theorem 5.2.4]), $\mathbb{D}$ is a finite-dimensional division algebra over $\mathbb{F}$, and thus $\mathbb{D}=\mathbb{F}$. Then the density theorem (cf. Kap72, Theorem 16, p. 95]) implies that

$$
U(\mathfrak{a}) / \operatorname{Ann}_{U(\mathfrak{a})}(S) \cong \operatorname{End}_{\mathbb{F}}(S) \cong \operatorname{Mat}_{d}(\mathbb{F}),
$$

where $d:=\operatorname{dim}_{\mathbb{F}} S$. Since $S$ is simple, $\operatorname{Ann}_{U(\mathfrak{a})}(S)$ is primitive (i.e., prime), and thus, $\operatorname{Ann}_{U(\mathfrak{a})}(S)$ is completely prime. It follows that $\operatorname{Mat}_{d}(\mathbb{F})$ has no zero divisors, i.e., $d=1$. Hence every simple $\mathfrak{a}-$ module is one-dimensional. By virtue of a result due to Jacobson, there exists a (finite-dimensional) faithful semisimple a-module (see [SF88, Theorem 5.5.2]). Therefore, we have

$$
[\mathfrak{a}, \mathfrak{a}] \subseteq \bigcap_{S \in \operatorname{Irr}(\mathfrak{a})} \operatorname{Ann}_{\mathfrak{a}}(S)=0
$$

where $\operatorname{Irr}(\mathfrak{a})$ denotes the set of isomorphism classes of simple $\mathfrak{a}$-modules, i.e., $\mathfrak{a}$ is abelian. Finally, the converse is just Mat58 Proposition 3.1].

Remark. The proof of Theorem 1.3 applied to a composition factor $S$ of the adjoint module of a finite-dimensional Lie algebra $\mathfrak{a}$ over a field of characteristic zero shows that in this case the universal enveloping algebra $U(\mathfrak{a})$ can only be a Matlis ring if $\mathfrak{a}$ is solvable (cf. also [BGR73, p. 49]). This still leaves the question as to whether Theorem 1.3 is also true in characteristic zero.

\section{Injective Hulls}

In this section several finiteness properties of injective hulls are considered. Let $R$ be an associative ring and let $M$ be a left or right $R$-module. An injective module $I$ is called an injective hull (or an injective envelope) of $M$ if there exists an $R$-module monomorphism $\iota: M \rightarrow I$ such that the image $\operatorname{Im}(\iota)$ of $\iota$ is an essential submodule of $I$. (By abuse of language, the pair $(I, \iota)$ is also called an injective hull of $M$.)

It is well-known that every module has an injective hull (cf. [GW89, Theorem 4.8(a)]). Moreover, injective hulls satisfy the following universal properties (cf. GW89 Theorem 4.8(b) and (c)] or [Rot79, Theorem 3.30]):

Let $M$ be an $R$-module and let $\left(I_{R}(M), \iota_{M}\right)$ be a injective hull of $M$.

(I) If $I$ is an injective $R$-module and $\iota$ is an $R$-module monomorphism from $M$ into $I$, then every $R$-module homomorphism $\eta$ from $I_{R}(M)$ into $I$ with $\eta \circ \iota_{M}=\iota$ is a monomorphism. (Since $I$ is injective and $\iota_{M}$ is an $R$-module monomorphism, there always exists an $R$-module homomorphism from $I_{R}(M)$ into $I$ with $\eta \circ \iota_{M}=\iota$ !)

(E) If $N$ is an $R$-module and $\varphi$ is an $R$-module monomorphism from $M$ into $N$, then every $R$ module homomorphism $\nu$ from $N$ into $I_{R}(M)$ with $\nu \circ \varphi=\iota_{M}$ is a monomorphism. (Since $I_{R}(M)$ is injective and $\varphi$ is an $R$-module monomorphism, there always exists an $R$-module homomorphism from $N$ into $I_{R}(M)$ with $\nu \circ \varphi=\iota_{M}$ !) 
(I) says that injective hulls are minimal injective extensions and (E) says that injective hulls are maximal essential extensions. In particular, injective hulls are uniquely determined up to isomorphism (cf. [GW89, Proposition 4.9]).

Recall that a module is said to be locally finite if every finitely generated (or equivalently, every cyclic) submodule is finite-dimensional.

Theorem 2.1 Let $\mathfrak{a}$ be a finite-dimensional Lie algebra over a field of prime characteristic. Then every essential extension of a locally finite $\mathfrak{a}$-module is locally finite.

Proof. Let $M$ be a locally finite a-module, let $E$ be an essential extension of $M$, and let $e$ be any non-zero element of $E$. Then $E^{\prime}:=U(\mathfrak{a}) e$ is an essential extension of $M^{\prime}:=E^{\prime} \cap M$. Since $U(\mathfrak{a})$ is noetherian, $M^{\prime} \subseteq E^{\prime}$ is finitely generated. Because $M$ is by assumption locally finite, $M^{\prime} \subseteq M$ is finite-dimensional. By virtue of Theorem [1.2 we can apply [Jat74 Corollary 3.6] or the main result of [Sch75] which both show that $E^{\prime}$ is also finite-dimensional, i.e., $E$ is locally finite.

The next result is an immediate consequence of Theorem 2.1

Corollary 2.2 If $\mathfrak{a}$ is a finite-dimensional Lie algebra over a field of prime characteristic, then the injective hull of every locally finite $\mathfrak{a}$-module is locally finite.

It is well-known that Corollary 2.2 is also true for a finite-dimensional solvable Lie algebra over an arbitrary field of characteristic zero (see [Don82, Theorem 2.2.3] and [Dah84. Corollary 12]), but it does not hold for a finite-dimensional semisimple Lie algebra over a field of characteristic zero (see Don82, Remark after the proof of Proposition 2.2.2] and [Dah89, Remark 1]). More precisely, we have the following result.

Theorem 2.3 Let $\mathfrak{a}$ be a finite-dimensional Lie algebra over a field of characteristic zero. Then the following statements are equivalent:

(1) $\mathfrak{a}$ is solvable.

(2) The injective hull of the one-dimensional trivial $\mathfrak{a}$-module is locally finite.

(3) The injective hull of every locally finite $\mathfrak{a}$-module is locally finite.

Proof. The implication $(1) \Longrightarrow(3)$ is just Don82, Theorem 2.2.3] or [Dah84 Corollary 12] and the implication $(3) \Longrightarrow(2)$ is trivial. Hence it only remains to show the implication $(2) \Longrightarrow(1)$.

Suppose that the injective hull $I_{\mathfrak{a}}(\mathbb{F})$ of the one-dimensional trivial a-module $\mathbb{F}$ is locally finite. Since the ground field is assumed to have characteristic zero, the Levi decomposition theorem (cf. Jac79, p. 91]) yields the existence of a semisimple subalgebra $\mathfrak{s}$ of $\mathfrak{a}$ (a so-called Levi factor of $\mathfrak{a})$ such that $\mathfrak{a}$ is the semidirect product of $\mathfrak{s}$ and its solvable radical $\operatorname{Solv}(\mathfrak{a})$. According to Dah84, Proposition 4], the restriction $I:=I_{\mathfrak{a}}(\mathbb{F})_{\mid \mathfrak{s}}$ is an injective $U(\mathfrak{s})$-module. Since $I_{\mathfrak{a}}(\mathbb{F})$ is a locally finite $U(\mathfrak{a})$-module, $I$ is a locally finite $U(\mathfrak{s})$-module.

Since $I$ is injective, it follows from the universal property (I) of injective hulls that $\mathbb{F} \subseteq I_{\mathfrak{s}}(\mathbb{F}) \subseteq I$. If $0 \neq m \in I_{\mathfrak{s}}(\mathbb{F})$, then the cyclic submodule $M:=U(\mathfrak{s}) m$ of $I$ is finite-dimensional. Since $I_{\mathfrak{s}}(\mathbb{F})$ is an essential extension of $\mathbb{F}$ and $M$ is a non-zero submodule of $I_{\mathfrak{s}}(\mathbb{F}), M \cap \mathbb{F} \neq 0$. Then for dimension reasons, $M \cap \mathbb{F}=\mathbb{F}$, i.e., $\mathbb{F} \subseteq M$. By virtue of Weyl's completely reducibility theorem (cf. Jac79] Theorem III.8, p. 79]), $\mathbb{F}$ has a complement in $M$, i.e., there exists a submodule $C$ of $M$ such that $M=\mathbb{F} \oplus C$. In particular, $\mathbb{F} \cap C=0$ which implies that $C=0$ because $C$ is a submodule of $I_{\mathfrak{s}}(\mathbb{F})$. Consequently, $M=\mathbb{F}$ and therefore $\mathbb{F}=I_{\mathfrak{s}}(\mathbb{F})$. Hence $\mathbb{F}$ is an injective $U(\mathfrak{s})$-module and 
thus also an injective $U(\mathbb{F} s)$-module for every element $s \in \mathfrak{s}$ (cf. Dah84, Proposition 4]). Finally $\operatorname{Ext}_{U(\mathbb{F} s)}^{1}(\mathbb{F}, \mathbb{F}) \cong H^{1}(\mathbb{F} s, \mathbb{F}) \neq 0$ for every $0 \neq s \in \mathfrak{s}$ yields $\mathfrak{s}=0$, i.e., $\mathfrak{a}=\operatorname{Solv}(\mathfrak{a})$ is solvable.

Let $\mathfrak{a}$ be a finite-dimensional Lie algebra over a field of characteristic zero. Donkin Don82, Theorem 2.2.3] proved that the largest locally finite submodule $I_{\mathfrak{a}}(M)_{\text {loc }}$ of the injective hull of any finite-dimensional $\mathfrak{a}$-module $M$ is artinian. In particular, if $\mathfrak{a}$ is solvable, then injective hulls of finite-dimensional a-modules are artinian. Furthermore, Dahlberg [Dah89] showed that the injective hull of every artinian $\mathfrak{s l}_{2}(\mathbb{C})$-module is locally artinian. In prime characteristic the following stronger result holds.

Theorem 2.4 If $\mathfrak{a}$ is a finite-dimensional Lie algebra over a field of prime characteristic, then the injective hull of every artinian $\mathfrak{a}$-module is artinian.

Proof. Let $M$ be an artinian $\mathfrak{a}$-module. Then the socle $\operatorname{Soc}_{\mathfrak{a}}(M)$ of $M$ is also artinian, i.e., a finite direct sum of simple modules. According to $I_{\mathfrak{a}}(M) \cong I_{\mathfrak{a}}\left(\operatorname{Soc}_{\mathfrak{a}}(M)\right)$ and the additivity of $I_{\mathfrak{a}}(-)$, the assertion is an immediate consequence of (PI) and [Jat75, Theorem 2].

Non-zero noetherian a-modules are very often not injective. This was proved in BHM82, Corollary 2.3] for every (not necessarily commutative) local noetherian associative ring and motivated the first part of Proposition 2.5 below. In particular, injective hulls of noetherian (or even finitedimensional) a-modules are never noetherian. Moreover, for artinian and locally finite a-modules any possible injective dimension can occur.

Proposition 2.5 Let $\mathfrak{a}$ be a finite-dimensional Lie algebra over a field $\mathbb{F}$ of prime characteristic. Then the following statements hold:

(1) For every non-zero finitely generated (= noetherian) a-module $M$, we have

$$
\text { inj. } \operatorname{dim}_{U(\mathfrak{a})} M=\operatorname{dim}_{\mathbb{F}} \mathfrak{a} .
$$

(2) For every integer $0 \leq r \leq \operatorname{dim}_{\mathbb{F}} \mathfrak{a}$ there exists an artinian $\mathfrak{a}$-module $M_{r}$ such that

$$
\text { inj. } \operatorname{dim}_{U(\mathfrak{a})} M_{r}=r .
$$

(3) For every integer $0 \leq r \leq \operatorname{dim}_{\mathbb{F}} \mathfrak{a}$ there exists a locally finite $\mathfrak{a}$-module $N_{r}$ such that

$$
\text { inj. } \operatorname{dim}_{U(\mathfrak{a})} N_{r}=r \text {. }
$$

Proof. (1): Since $M$ is noetherian, it has a maximal submodule $N$. Hence $S:=M / N$ is simple and thus finite-dimensional (cf. [SF88, Theorem 5.2.4]). By virtue of [Far90 Theorem 4.2(3)], there exists an $\mathfrak{a}$-module $V$ such that $\operatorname{Ext}_{U(\mathfrak{a})}^{d}(V, S) \neq 0$, where $d:=\operatorname{dim}_{\mathbb{F}} \mathfrak{a}$. Then the long exact cohomology sequence implies the exactness of

$$
\operatorname{Ext}_{U(\mathfrak{a})}^{d}(V, M) \longrightarrow \operatorname{Ext}_{U(\mathfrak{a})}^{d}(V, S) \longrightarrow \operatorname{Ext}_{U(\mathfrak{a})}^{d+1}(V, N) .
$$

Because of gl.dim $U(\mathfrak{a})=d$ (cf. [CE56. Theorem 8.2]), the right-hand term vanishes. One concludes that $\operatorname{Ext}_{U(\mathfrak{a})}^{d}(V, M) \neq 0$, i.e., inj. $\operatorname{dim}_{U(\mathfrak{a})} M \geq d$. The reverse inequality follows from inj. $\operatorname{dim}_{U(\mathfrak{a})} M \leq$ gl.dim $U(\mathfrak{a})=d$.

(2): Put $d:=\operatorname{dim}_{\mathbb{F}} \mathfrak{a}$ and let $M_{d}$ be any finite-dimensional non-zero a-module. By the first part, we have inj. $\operatorname{dim}_{U(\mathfrak{a})} M_{d}=d$. According to Theorem 2.4 the injective hull $I_{\mathfrak{a}}\left(M_{d}\right)$ and therefore 
$M_{d-1}:=I_{\mathfrak{a}}\left(M_{d}\right) / M_{d}$ are artinian. From the long exact cohomology sequence and the injectivity of $I_{\mathfrak{a}}\left(M_{d}\right)$ one concludes for an arbitrary $\mathfrak{a}$-module $X$ that

$$
\operatorname{Ext}_{U(\mathfrak{a})}^{d}\left(X, M_{d-1}\right) \cong \operatorname{Ext}_{U(\mathfrak{a})}^{d+1}\left(X, M_{d}\right)=0
$$

because inj. $\operatorname{dim}_{U(\mathfrak{a})} M_{d}=d$. Hence inj.dim $\operatorname{dia}_{U(\mathfrak{a})} M_{d-1} \leq d-1$ (cf. Rot79 Theorem 9.8]). By another application of [Rot79, Theorem 9.8], there exists an a-module $X_{d}$ such that $\operatorname{Ext}_{U(\mathfrak{a})}^{d}\left(X_{d}, M_{d}\right) \neq 0$. Then the long exact cohomology sequence implies

$$
\operatorname{Ext}_{U(\mathfrak{a})}^{d-1}\left(X_{d}, M_{d-1}\right) \cong \operatorname{Ext}_{U(\mathfrak{a})}^{d}\left(X_{d}, M_{d}\right) \neq 0,
$$

i.e., inj. $\operatorname{dim}_{U(\mathfrak{a})} M_{d-1}=d-1$, and the assertion follows by induction.

(3): The proof is the same as for (2) except that one uses Corollary 2.2 instead of Theorem 2.4 to conclude that $N_{d-1}:=I_{\mathfrak{a}}\left(N_{d}\right) / N_{d}$ is locally finite.

Remark. Dually, non-zero artinian a-modules are never projective and for noetherian a-modules any possible projective dimension can occur (see [Fel90]).

Since every simple module over a finite-dimensional Lie algebra over a field of prime characteristic is finite-dimensional (cf. [SF88, Theorem 5.2.4], the following is an immediate consequence of Proposition 2.5 (1).

Corollary 2.6 Let $\mathfrak{a}$ be a finite-dimensional Lie algebra over a field $\mathbb{F}$ of prime characteristic and let $S$ be a simple $\mathfrak{a}$-module. Then inj. $\operatorname{dim}_{U(\mathfrak{a})} S=\operatorname{dim}_{\mathbb{F}} \mathfrak{a}$.

\section{Locally Finite Submodules of the Coregular Module}

Let $\mathfrak{a}$ be a Lie algebra over a field $\mathbb{F}$ of arbitrary characteristic. Then the linear dual $U(\mathfrak{a})^{*}:=$ $\operatorname{Hom}_{\mathbb{F}}(U(\mathfrak{a}), \mathbb{F})$ of $U(\mathfrak{a})$ is a left and a right $U(\mathfrak{a})$-module, the so-called coregular module of $U(\mathfrak{a})$ (cf. [Dix96, 2.7.7]). It is well-known that $U(\mathfrak{a})^{*}$ is injective as a left and right $U(\mathfrak{a})$-module (cf. [Lev76] Proposition 1]).

Let $U(\mathfrak{a})^{\circ}$ denote the continuous dual of $U(\mathfrak{a})$ which is the largest locally finite submodule of the left and right $U(\mathfrak{a})$-module $U(\mathfrak{a})^{*}$. It is well-known that $U(\mathfrak{a})^{\circ}$ also consists of all linear forms on $U(\mathfrak{a})$ that vanish on some two-sided ideal of finite codimension in $U(\mathfrak{a})$ (cf. [Lev86, p. 51]).

Finally, let $U(\mathfrak{a})^{\natural}$ denote the set of all linear forms on $U(\mathfrak{a})$ that vanish on a certain power of the augmentation ideal $U(\mathfrak{a})_{+}$of $U(\mathfrak{a})$. Then one has the following inclusions where $\mathbb{F}^{*}$ is identified with the linear forms on $U(\mathfrak{a})$ that vanish on $U(\mathfrak{a})_{+}$(cf. Dix96, Lemma 2.5.1]):

$$
\mathbb{F} \cong \mathbb{F}^{*} \subseteq U(\mathfrak{a})^{\natural} \subseteq U(\mathfrak{a})^{\circ} \subseteq U(\mathfrak{a})^{*} .
$$

If $\mathfrak{a} \neq 0$, then all these inclusions are proper.

The following is also well-known (cf. [Lev76, Lemme 2]).

Lemma 3.1 If $\mathfrak{a}$ is a finite-dimensional Lie algebra over an arbitrary field, then $U(\mathfrak{a})^{\natural}$ is an essential extension of the one-dimensional trivial left and right $U(\mathfrak{a})$-module.

For the convenience of the reader we include a proof of the following result.

Theorem 3.2 (cf. [Lev76, Théorème 3] or Dah84, Theorem 3]) If a is a finite-dimensional nilpotent Lie algebra over an arbitrary field, then $U(\mathfrak{a})^{\natural}$ is an injective hull of the one-dimensional trivial left and right $U(\mathfrak{a})$-module. 
Proof. Since $\mathbb{F} \cong \mathbb{F}^{*} \subseteq U(\mathfrak{a})^{*}$ and $U(\mathfrak{a})^{*}$ is injective, the universal property (I) of injective hulls implies that $I_{\mathfrak{a}}(\mathbb{F}) \subseteq U(\mathfrak{a})^{*}$. It follows from Dah84 Proposition 1] that $I_{\mathfrak{a}}(\mathbb{F})$ is locally finite. Consider $\varphi \in I_{\mathfrak{a}}(\mathbb{F})$. Then $E:=U(\mathfrak{a}) \varphi$ is a finite-dimensional extension of $\mathbb{F}$. An application of Fitting's lemma (cf. Jac79 Theorem II.4, p. 39]) shows that $\mathfrak{a}$ acts nilpotently on $E$ and it follows from the Engel-Jacobson theorem (cf. [SF88, Corollary 1.3.2]) that a certain power of the augmentation ideal $U(\mathfrak{a})_{+}$annihilates $E$. Consequently, $\varphi \in U(\mathfrak{a})^{\natural}$ and therefore $I_{\mathfrak{a}}(\mathbb{F}) \subseteq U(\mathfrak{a})^{\natural}$. Finally, the other inclusion follows from Lemma 3.1 and the universal property (E) of injective hulls.

Remark. It is observed in [Lev76, Remarque 2 after Théorème 3] that $U(\mathfrak{a})^{\natural}$ is not injective for the two-dimensional non-nilpotent Lie algebra. It would be interesting to know whether the injectivity of $U(\mathfrak{a})^{\natural}$ implies that $\mathfrak{a}$ is nilpotent.

The isomorphism $H^{n}\left(\mathfrak{a}, U(\mathfrak{a})^{\mathfrak{a}}\right) \cong \operatorname{Ext}_{U(\mathfrak{a})}^{n}\left(\mathbb{F}, U(\mathfrak{a})^{\mathfrak{\natural}}\right)$ in conjunction with Theorem 3.2 and Rot79, Theorem 7.6] yields the following cohomological vanishing theorem due to Koszul:

Corollary 3.3 (cf. Kos54, Théorème 6]) If $\mathfrak{a}$ is a finite-dimensional nilpotent Lie algebra over an arbitrary field, then

$$
H^{n}\left(\mathfrak{a}, U(\mathfrak{a})^{\natural}\right)=0
$$

for every positive integer $n$.

Question. Does the vanishing $H^{n}\left(\mathfrak{a}, U(\mathfrak{a})^{\natural}\right)$ for every positive integer $n$ imply that $\mathfrak{a}$ is nilpotent?

Let us now consider arbitrary finite-dimensional Lie algebras over fields of prime characteristic.

Theorem 3.4 If $\mathfrak{a}$ is a finite-dimensional Lie algebra over a field of prime characteristic, then the continuous dual $U(\mathfrak{a})^{\circ}$ is injective as a left and right $U(\mathfrak{a})$-module.

Proof. Since $U(\mathfrak{a})^{\circ} \subseteq U(\mathfrak{a})^{*}$ and $U(\mathfrak{a})^{*}$ is injective, the universal property (I) of injective hulls implies that $I_{\mathfrak{a}}\left(U(\mathfrak{a})^{\circ}\right) \subseteq U(\mathfrak{a})^{*}$. Because $U(\mathfrak{a})^{\circ}$ is locally finite, it follows from Corollary 2.2 that $I_{\mathfrak{a}}\left(U(\mathfrak{a})^{\circ}\right)$ is also locally finite. But since by definition $U(\mathfrak{a})^{\circ}$ is the largest locally finite submodule of $U(\mathfrak{a})^{*}, U(\mathfrak{a})^{\circ}=I_{\mathfrak{a}}\left(U(\mathfrak{a})^{\circ}\right)$ is injective.

The isomorphism $H^{n}\left(\mathfrak{a}, U(\mathfrak{a})^{\circ}\right) \cong \operatorname{Ext}_{U(\mathfrak{a})}^{n}\left(\mathbb{F}, U(\mathfrak{a})^{\circ}\right)$ in conjunction with Theorem 3.4 and Rot79 Theorem 7.6] yields the following cohomological vanishing theorem:

Corollary 3.5 If $\mathfrak{a}$ is a finite-dimensional Lie algebra over a field of prime characteristic, then

$$
H^{n}\left(\mathfrak{a}, U(\mathfrak{a})^{\circ}\right)=0
$$

for every positive integer $n$.

Remark. The case $n=1$ of Corollary 3.5 was already proved by Masuoka [Mas00, Proposition 5.1]. It follows from Corollary [3.5] in conjunction with [Kos54, Théorème 2] that every cohomology class of a finite-dimensional Lie algebra over a field of prime characteristic with coefficients in a finite-dimensional module is annihilable. This result was proved in a completely different way by Dzumadil'daev [Dzu90, Theorem 3.1, pp. 467-470].

The equivalence of (1), (3), and (4) in the next result is essentially due to Koszul (see [Kos54, Théorème 7 and p. 536]. Moreover, for an algebraically closed ground field the implication $(1) \Longrightarrow(2)$ follows from BM85. Théorème 3.6 and Théorème 4.10] (see also Lev86, Proposition 3.4 and Proposition 3.6] for $\mathbb{F}=\mathbb{C})$. 
Theorem 3.6 Let $\mathfrak{a}$ be a finite-dimensional Lie algebra over a field of characteristic zero. Then the following statements are equivalent:

(1) $\mathfrak{a}$ is solvable.

(2) The continuous dual $U(\mathfrak{a})^{\circ}$ is injective as a left and right $U(\mathfrak{a})$-module.

(3) $H^{n}\left(\mathfrak{a}, U(\mathfrak{a})^{\circ}\right)=0$ for every positive integer $n$.

(4) $H^{3}\left(\mathfrak{a}, U(\mathfrak{a})^{\circ}\right)=0$.

Proof. The proof of the implication $(1) \Longrightarrow(2)$ is the same as for Theorem 3.4 except that one uses Theorem [2.3 instead of Corollary [2.2] in order to conclude that $I_{\mathfrak{a}}\left(U(\mathfrak{a})^{\circ}\right)$ is locally finite. Since $(2) \Longrightarrow(3)$ is clear and (4) is just a special case of (3), it remains to show the implication $(4) \Longrightarrow(1)$.

Suppose that $H^{3}\left(\mathfrak{a}, U(\mathfrak{a})^{\circ}\right)=0$ and let $M$ be an arbitrary finite-dimensional $\mathfrak{a}$-module. Then the isomorphism $\operatorname{Hom}_{\mathbb{F}}(U(\mathfrak{a}), M) \cong U(\mathfrak{a})^{*} \otimes_{\mathbb{F}} M$ (where $M$ is considered as a trivial a-module) implies that $H^{3}\left(\mathfrak{a}, \operatorname{Hom}_{\mathbb{F}}(U(\mathfrak{a}), M)_{\text {loc }}\right)=0$ where $\operatorname{Hom}_{\mathbb{F}}(U(\mathfrak{a}), M)_{\text {loc }}$ denotes the largest locally finite submodule of $\operatorname{Hom}_{\mathbb{F}}(U(\mathfrak{a}), M)$. According to $\operatorname{Kos} 54$, Théorème 2], it follows that every cohomology class in $H^{3}(\mathfrak{a}, M)$ is annihilable and thus [Kos54 5), p. 536] yields that $\mathfrak{a}$ is solvable.

Remark. The above proof of the implication $(1) \Longrightarrow(2)$ is not only much more direct than in BM85. or Lev86 but also answers affirmatively a question posed at the end of the third section in BM85. Moreover, it should be noted that the implication $(2) \Longrightarrow(1)$ in Theorem [3.6] can also be obtained directly from the universal property (I) of injective hulls and Theorem 2.3

Recently, H.-J. Schneider has generalized the implication $(1) \Longrightarrow(2)$ in Theorem 3.6 even further. Let $\mathfrak{a}$ be a finite-dimensional Lie algebra over a field of characteristic zero and let $\operatorname{Solv}(\mathfrak{a})$ denote the solvable radical of $\mathfrak{a}$. Then Schneider proves that the restriction $\left[U(\mathfrak{a})^{\circ}\right]_{\mid \operatorname{Solv}(\mathfrak{a})}$ of $U(\mathfrak{a})^{\circ}$ to $\operatorname{Solv}(\mathfrak{a})$ is injective (cf. Mas00 Theorem 5.3]). This in conjunction with the Hochschild-Serre spectral sequence (cf. [HS53, Theorem 6]) and the two Whitehead lemmata (cf. [Jac79, Theorem III.13]) implies that $H^{1}\left(\mathfrak{a}, U(\mathfrak{a})^{\circ}\right)=0=H^{2}\left(\mathfrak{a}, U(\mathfrak{a})^{\circ}\right)$ (see Mas00, Proposition 5.1 and Theorem 5.2]). But Theorem 3.6 shows that $H^{3}\left(\mathfrak{a}, U(\mathfrak{a})^{\circ}\right) \neq 0$ if $\mathfrak{a}$ is not solvable which generalizes [Mas00, Remark 5.9].

It follows from the universal properties (E) and (I) of injective hulls in conjunction with Lemma 3.1 and Theorem 3.4 that

$$
\mathbb{F} \cong \mathbb{F}^{*} \subseteq U(\mathfrak{a})^{\natural} \subseteq I_{\mathfrak{a}}(\mathbb{F}) \subseteq U(\mathfrak{a})^{\circ} .
$$

Note that the cocommutative Hopf algebra structure on $U(\mathfrak{a})$ induces a commutative Hopf algebra structure on $U(\mathfrak{a})^{*}$ which as an $\mathbb{F}$-algebra can be identified with the algebra of power series in $\operatorname{dim}_{\mathbb{F}} \mathfrak{a}$ variables (cf. [Dix96, Proposition 2.7.5]) and the continuous dual $U(\mathfrak{a})^{\circ}$ is a subalgebra of $U(\mathfrak{a})^{*}$.

Let $\mathfrak{a}$ be a finite-dimensional solvable Lie algebra over the complex numbers. Then Levasseur Lev86. Théorème 2.2] has shown that $I_{\mathfrak{a}}(\mathbb{F})$ is isomorphic to a polynomial algebra in $\operatorname{dim}_{\mathbb{F}} \mathfrak{a}$ variables on which $\mathfrak{a}$ acts via derivations.

Conjecture. Let $\mathfrak{a}$ be a finite-dimensional Lie algebra over a field $\mathbb{F}$. If $\mathfrak{a}$ is solvable and $\operatorname{char}(\mathbb{F})=0$ or if $\mathfrak{a}$ is arbitrary and $\operatorname{char}(\mathbb{F})>0$, then $I_{\mathfrak{a}}(\mathbb{F})$ is isomorphic to a polynomial algebra in $\operatorname{dim}_{\mathbb{F}} \mathfrak{a}$ variables on which $\mathfrak{a}$ acts via derivations.

If $\mathfrak{a}$ is abelian, then this follows from [Nor74, Theorem 2] and in Dah84, Section 4] there are examples confirming this for Lie algebras of small dimensions. 


\section{Minimal Injective Resolutions}

Let $\mathcal{I}$ be a two-sided ideal of an associative ring $R$. Then

$$
\text { u.gr( } \mathcal{I}):=\sup \left\{n \in \mathbb{N}_{0} \mid \operatorname{Ext}_{R}^{n}(R / \mathcal{I}, R) \neq 0\right\}
$$

and

$$
\operatorname{logr}(\mathcal{I}):=\inf \left\{n \in \mathbb{N}_{0} \mid \operatorname{Ext}_{R}^{n}(R / \mathcal{I}, R) \neq 0\right\}
$$

are called upper grade and lower (or homological) grade of $\mathcal{I}$, respectively. A left and right noetherian associative ring $R$ is left (resp. right) injectively homogeneous over a central subring $C$ if $R$ is integral over $C$, inj. $\operatorname{dim}_{R} R<\infty$ (resp. inj. $\left.\operatorname{dim} R_{R}<\infty\right)$ and u.gr $(\mathcal{M})=u \cdot \operatorname{gr}(\mathcal{N})$ for all maximal ideals $\mathcal{M}$ and $\mathcal{N}$ such that $\mathcal{M} \cap C=\mathcal{N} \cap C$. In [BH88] it was demonstrated that for associative rings integral over a central subring the class of injectively homogeneous rings is a natural generalization of the class of commutative Gorenstein rings. Moreover, $\mathrm{BH} 88$. Corollary 3.6] shows that $R$ is injectively homogeneous over its center $C(R)$ if and only if $R$ is injectively homogeneous over every subring $C \subseteq C(R)$ over which $R$ is integral, and by virtue of [BH88 Corollary 4.4], $R$ is left injectively homogeneous if and only if $R$ is right injectively homogeneous.

Lemma 4.1 If $\mathfrak{a}$ is an finite-dimensional Lie algebra over a field of prime characteristic, then $U(\mathfrak{a})$ is injectively homogeneous over every subring $C$ of $U(\mathfrak{a})$ with $\mathcal{O}(\mathfrak{a}) \subseteq C \subseteq C(U(\mathfrak{a}))$.

Proof. Let $\mathcal{M}$ be a maximal ideal of $U(\mathfrak{a})$. Then $\wp:=\mathcal{M} \cap C(U(\mathfrak{a}))$ is also maximal SF88 Corollary 6.3.4], and thus Hilbert's Nullstellensatz yields that $C(U(\mathfrak{a})) / \wp$ is finite-dimensional. Since $U(\mathfrak{a})$ is finitely generated over $C(U(\mathfrak{a}))$, we conclude that $M:=U(\mathfrak{a}) / \mathcal{M}$ is also finite-dimensional. Set $d:=\operatorname{dim}_{\mathbb{F}} \mathfrak{a}$. According to [Far90, Theorem 4.2(3)], there exists a simple a-module $S$ such that $\operatorname{Ext}_{U(\mathfrak{a})}^{d}(M, S) \neq 0$. If $\mathcal{A}$ denotes the annihilator of a generator of $S$ in $U(\mathfrak{a})$, we obtain a short exact sequence $0 \rightarrow \mathcal{A} \rightarrow U(\mathfrak{a}) \rightarrow S \rightarrow 0$ of $U(\mathfrak{a})$-modules. The long exact cohomology sequence implies the exactness of

$$
\operatorname{Ext}_{U(\mathfrak{a})}^{d}(M, U(\mathfrak{a})) \longrightarrow \operatorname{Ext}_{U(\mathfrak{a})}^{d}(M, S) \longrightarrow \operatorname{Ext}_{U(\mathfrak{a})}^{d+1}(M, \mathcal{A}) .
$$

Because of gl.dim $U(\mathfrak{a})=d$ (cf. CE56 Theorem 8.2]), the right-hand term vanishes. We conclude that $\operatorname{Ext}_{U(\mathfrak{a})}^{d}(M, U(\mathfrak{a})) \neq 0$, i.e., $\operatorname{u.gr}(\mathcal{M}) \geq \operatorname{logr}(\mathcal{M}) \geq d$. The reverse inequality follows from u.gr $(\mathcal{M}) \leq$ gl.dim $U(\mathfrak{a})=d$. Hence u.gr $(\mathcal{M})=d$ for every maximal ideal of $U(\mathfrak{a})$. This and (IC) yield the assertion.

Remark. Let $\mathfrak{a}$ be an finite-dimensional Lie algebra over a field of characteristic zero. According to a theorem of Latyšev [Lat63, $U(\mathfrak{a})$ is a PI algebra if and only if $\mathfrak{a}$ is abelian. Since every algebra which is a finitely generated module over its center is a PI algebra (cf. [GW89 p. xi]), $U(\mathfrak{a})$ is injectively homogeneous over its center if and only if $\mathfrak{a}$ is abelian.

One consequence of Lemma 4.1 is that inj. $\operatorname{dim} U(\mathfrak{a})_{\wp}<\infty$ for every semiprime ideal $\wp$ of every subring $C$ of $U(\mathfrak{a})$ with $\mathcal{O}(\mathfrak{a}) \subseteq C \subseteq C(U(\mathfrak{a})$ ) (cf. [Bas63, Fundamental Theorem (e), p. 10] and [BH88, Theorem 4.1]). More importantly for the purpose of this paper, it is an immediate consequence of Lemma 4.1] and [BH88, Theorem 5.5] that the minimal injective resolution of $U(\mathfrak{a})$ has the same form as for commutative Gorenstein rings (cf. Bas63, Fundamental Theorem (f), p. 10]). Recall that a minimal injective resolution of a module $M$ is a long excact sequence

$$
0 \longrightarrow M \longrightarrow I_{0} \stackrel{d_{0}}{\longrightarrow} \cdots \longrightarrow I_{n} \stackrel{d_{0}}{\longrightarrow} \cdots
$$

such that $I_{n}$ is an injective hull of $\operatorname{Ker}\left(d_{n}\right)$ for every non-negative integer $n$. 
Theorem 4.2 Let $\mathfrak{a}$ be an finite-dimensional Lie algebra over a field $\mathbb{F}$ of prime characteristic. If $0 \longrightarrow U(\mathfrak{a}) \longrightarrow I_{0} \longrightarrow \cdots \longrightarrow I_{d} \longrightarrow 0$ is a minimal injective resolution of $U(\mathfrak{a})$ as a left or right $U(\mathfrak{a})$-module, then

$$
I_{n} \cong \bigoplus_{\operatorname{ht}(\mathcal{P})=n} I_{\mathfrak{a}}(U(\mathfrak{a}) / \mathcal{P})
$$

for every $0 \leq n \leq d:=\operatorname{dim}_{\mathbb{F}} \mathfrak{a}$.

Remark. If $\mathfrak{a}$ is a finite-dimensional Lie algebra over a field of characteristic zero, then the structure of a minimal injective resolution of $U(\mathfrak{a})$ is even in the solvable case more complicated than in Theorem 4.2 (cf. Mal83, Mal86]).

Let $\mathfrak{a}$ be a finite-dimensional solvable Lie algebra over an algebraically closed field of characteristic zero. Then the last term of a minimal injective resolution of $U(\mathfrak{a})$ is isomorphic to the continuous dual $U(\mathfrak{a})^{\circ}$ of $U(\mathfrak{a})$ (see BM85, Théorème 3.6 and Théorème 4.10] and also Lev86. Proposition 3.4 and Proposition 3.6] for $\mathbb{F}=\mathbb{C}$ ). We conclude the paper by applying Theorem 4.2 in order to prove the analogue of this result in prime characteristic.

Theorem 4.3 Let $\mathfrak{a}$ be a finite-dimensional Lie algebra over an algebraically closed field of prime characteristic. If $0 \longrightarrow U(\mathfrak{a}) \longrightarrow I_{0} \longrightarrow \cdots \longrightarrow I_{d} \longrightarrow 0$ is a minimal injective resolution of $U(\mathfrak{a})$ as a left or right $U(\mathfrak{a})$-module, then $I_{d} \cong U(\mathfrak{a})^{\circ}$.

Proof. By virtue of Corollary 2.2 injective hulls of locally finite modules are locally finite. Since $\mathbb{F}$ is algebraically closed, this enables one to prove that

$$
U(\mathfrak{a})^{\circ} \cong \bigoplus_{S \in \operatorname{Irr}(\mathfrak{a})} I_{\mathfrak{a}}(S)^{\oplus \operatorname{dim}_{\mathbb{F}} S}
$$

as left or right $U(\mathfrak{a})$-module, where $\operatorname{Irr}(\mathfrak{a})$ denotes the set of isomorphism classes of simple $\mathfrak{a}$-modules (cf. [Gre76, 1.5] for the analogous statement in terms of coalgebras and comodules). On the other hand, it follows from (PI) and [Sch76, Theorem 4] that a prime ideal $\mathcal{P}$ of $U(\mathfrak{a})$ has maximal height $d$ if and only if $\mathcal{P}$ is maximal. But every maximal ideal $\mathcal{P}$ of $U(\mathfrak{a})$ is primitive, i.e., there is a simple $\mathfrak{a}$-module $S$ such that $\mathcal{P}=\operatorname{Ann}_{U(\mathfrak{a})}(S)$. Hence

$$
U(\mathfrak{a}) / \mathcal{P}=U(\mathfrak{a}) / \operatorname{Ann}_{U(\mathfrak{a})}(S) \cong \operatorname{End}_{\mathbb{F}}(S) \cong S^{\oplus \operatorname{dim}_{F} S^{*}}
$$

as a left or right $U(\mathfrak{a})$-module. According to (PI) and Kaplansky's theorem (cf. Kap72 Theorem $50]$ ), every primitive ideal of $U(\mathfrak{a})$ is maximal and therefore

$$
I_{d} \cong \bigoplus_{\operatorname{ht}(\mathcal{P})=d} I_{\mathfrak{a}}(U(\mathfrak{a}) / \mathcal{P}) \cong \bigoplus_{S \in \operatorname{Irr}(\mathfrak{a})} I_{\mathfrak{a}}(S)^{\oplus \operatorname{dim}_{\mathbb{F}} S^{*}} \cong U(\mathfrak{a})^{\circ}
$$

Question. Does Theorem 4.3 remain valid for arbitrary ground fields of prime characteristic?

\section{References}

[Bas63] H. Bass: On the ubiquity of Gorenstein rings, Math. Z. 82 (1963), 8-28. 
[BM85] G. Barou and M.-P. Malliavin: Sur la résolution injective minimale de l'algèbre enveloppante d'une algèbre de Lie résoluble, J. Pure Appl. Algebra 37 (1985), 1-25.

[BGR73] W. Borho, P. Gabriel, and R. Rentschler: Primideale in Einhüllenden auflösbarer LieAlgebren, Lecture Notes in Mathematics 357, Springer, Berlin/Heidelberg/New York, 1973.

[BH88] K. A. Brown and C. R. Hajarnavis: Injectively homogeneous rings, J. Pure Appl. Algebra 51 (1988), 65-77.

[BHM82] K. A. Brown, C. R. Hajarnavis, and A. B. MacEacharn: Noetherian rings of finite global dimension, Proc. London Math. Soc. (3) 44 (1982), 349-371.

[CE56] H. Cartan and S. Eilenberg: Homological Algebra, Princeton University Press, Princeton, New Jersey, 1956.

[Dah84] R. P. Dahlberg: Injective hulls of Lie modules, J. Algebra 87 (1984), 458-471.

[Dah89] R. P. Dahlberg: Injective hulls of simple sl(2,C)-modules are locally artinian, Proc. Amer. Math. Soc. 107 (1989), 35-37.

[Dix96] J. Dixmier: Enveloping Algebras, revised reprint of the 1977 translation, Graduate Studies in Mathematics 11, Amer. Math. Soc., Providence, RI, 1996.

[Don82] S. Donkin: On the Hopf algebra dual of an enveloping algebra, Math. Proc. Cambridge Philos. Soc. 91 (1982), 215-224.

[Dzu90] A. S. Dzumadil'daev: Cohomology of truncated coinduced representations of Lie algebras of positive characteristic, Math. USSR Sbornik 66 (1990), 461-473.

[Far90] R. Farnsteiner: Extension functors of modular Lie algebras, Math. Ann. 288 (1990), 713730 .

[Fel90] J. Feldvoss: Injective modules and the prime spectrum of universal enveloping algebras, unpublished manuscript, 1990.

[GW89] K. R. Goodearl and R. B. Warfield, Jr.: An Introduction to Noncommutative Noetherian Rings, London Mathematical Society Student Notes 16, Cambridge University Press, Cambridge, 1989.

[Gre76] J. A. Green: Locally finite representations, J. Algebra 41 (1976), 137-171.

[HS53] G. Hochschild and J.-P. Serre: Cohomology of Lie algebras, Ann. of Math. (2) 57 (1953), 591-603.

[Jac79] N. Jacobson: Lie Algebras, Dover Publications, Inc., New York, 1979 (unabridged and corrected republication of the original edition from 1962).

[Jat74] A. V. Jategaonkar: Jacobson's conjecture and modules over fully bounded noetherian rings, J. Algebra 30 (1974), 103-121.

[Jat75] A. V. Jategaonkar: Certain injectives are artinian, in: Noncommutative Ring Theory, Kent State, 1975 (eds. J. H. Cozzens and F. L. Sandomierski), Lecture Notes in Mathematics 545, Springer, Berlin/Heidelberg/New York, 1976, pp. 128-139. 
[Kap72] I. Kaplansky: Fields and Rings, 2nd edition, Chicago Lectures in Mathematics, The University of Chicago Press, Chicago/London, 1972.

[Kos54] J. L. Koszul: Sur les modules de représentation des algèbres de Lie résolubles, Amer. J. Math. 76 (1954), 535-554.

[Kra70] G. Krause: On the Krull-dimension of left noetherian left Matlis-rings, Math. Z. 118 (1970), 207-214.

[Kra72] G. Krause: On fully left bounded left noetherian rings, J. Algebra 23 (1972), 88-99.

[Lat63] V. N. Latyšev: Two remarks on PI-algebras (in Russian), Sibirsk. Mat. Ž. 4 (1963), $1120-1121$.

[Lev76] T. Levasseur: Cohomologie des algèbres de Lie nilpotentes et enveloppes injectives, Bull. Sci. Math. (2) 100 (1976), 377-383.

[Lev86] T. Levasseur: L'enveloppe injective du module trivial sur une algèbre de Lie résoluble, Bull. Sci. Math. (2) 110 (1986), 49-61.

[Mal83] M.-P. Malliavin: Modules sans torsion et modules injectifs sur les algèbres de Lie résolubles, J. Algebra 83 (1983), 126-157.

[Mal86] M.-P. Malliavin: Représentations injectives d'algèbres de Lie résolubles, Comm. Algebra 14 (1986), 1503-1513.

[Mat58] E. Matlis: Injective modules over noetherian rings, Pacific J. Math. 8 (1958), 511-528.

[Mas00] A. Masuoka: Extensions of Hopf algebras and Lie bialgebras, Trans. Amer. Math. Soc. 352 (2000), 3837-3879.

[Nor74] D. G. Northcott: Injective envelopes and inverse polynomials, J. London Math. Soc. (2) 8 (1974), 290-296.

[Rot79] J. J. Rotman: An Introduction to Homological Algebra, Academic Press, Inc., Boston/San Diego/New York, 1979.

[Sch75] W. Schelter: Essential extensions and intersection theorems, Proc. Amer. Math. Soc. 53 (1975), 328-330.

[Sch76] W. Schelter: Integral extensions of rings satisfying a polynomial identity, J. Algebra 40 (1976), 245-257.

[SF88] H. Strade and R. Farnsteiner: Modular Lie Algebras and Their Representations, Monographs and Textbooks in Pure and Applied Mathematics 116, Marcel Dekker, Inc., New York/Basel, 1988. 